García-Ceberino, J. M.; Antúnez, A.; Feu, S.; Ibáñez, S. J. (2020) Validation of Two Intervention Programs for Teaching School Soccer. Revista Internacional de Medicina y Ciencias de la Actividad Física y el Deporte vol. 20 (78) pp. 257-274 Http://cdeporte.rediris.es/revista/revista78/artvalidacion1155.htm DOI: http://doi.org/10.15366/rimcafd2020.78.005

\title{
ORIGINAL
}

\section{VALIDACIÓN DE DOS PROGRAMAS DE INTERVENCIÓN PARA LA ENSEÑANZA DEL FÚTBOL ESCOLAR}

\section{VALIDATION OF TWO INTERVENTION PROGRAMS FOR TEACHING SCHOOL SOCCER}

\author{
García-Ceberino, J.M. ${ }^{1,5}$; Antúnez, A. ${ }^{2,5}$; Feu, S. ${ }^{3,5}$ e Ibáñez, S.J. ${ }^{4,5}$ \\ ${ }^{1}$ Doctorando en Investigación en la Enseñanza y el Aprendizaje de las Ciencias Experimentales, \\ Sociales, Matemáticas y la Actividad Física y Deportiva. Universidades de Extremadura y Huelva \\ (España) jgarciaxp@alumnos.unex.es \\ 2 Profesor Contratado Doctor, Facultad de Ciencias del Deporte. Universidad de Extremadura \\ (España) antunez@unex.es \\ ${ }^{3}$ Profesor Titular de Universidad, Facultad de Educación. Universidad de Extremadura (España) \\ sfeu@unex.es \\ ${ }^{4}$ Catedrático de Universidad, Facultad de Ciencias del Deporte. Universidad de Extremadura \\ (España) sibanez@unex.es \\ ${ }^{5}$ GOERD. Grupo de Optimización del Entrenamiento y Rendimiento Deportivo.
}

\section{Agradecimientos}

Este trabajo ha sido parcialmente subvencionado por la Ayuda a los Grupos de Investigación (GR18170) del Gobierno de Extremadura (Consejería de Empleo, Empresa e Innovación); con la aportación de la Unión Europea a través de los Fondos Europeos de Desarrollo Regional.

Código UNESCO / UNESCO code: 5801 Teoría y métodos educativos / Theory and educational methods 5801.07 Métodos pedagógicos / Pedagogical methods. Clasificación Consejo de Europa / Council of Europe classification: 5. Didáctica y metodología / 5. Teaching and methodology.

Recibido 2 de julio de 2018 Received July 2, 2018

Aceptado 13 enero de 2019 Accepted January 13, 2019

\section{RESUMEN}

El propósito del estudio fue validar dos programas de intervención, correspondientes a dos unidades didácticas realizadas cada una en base a dos metodologías diferentes: Direct Instruction (ID) y Tactical Games Approach (TGA), para la enseñanza del fútbol escolar. Se diseñaron dos programas de intervención semejantes con el mismo número de tareas, sesiones, fases de 
juego, contenidos y objetivos. En el proceso de validación participaron 13 jueces expertos. La validez de contenido se calculó mediante el coeficiente de $V$ de Aiken y sus intervalos de confianza. Para la consistencia interna se empleó el coeficiente de a de Cronbach. Ninguna de las tareas que componen los programas de intervención fue eliminada al superar el valor crítico exacto $(V \geq$ .69). La consistencia interna de las tareas fue excelente $(\alpha=.97)$. Por tanto, ambos programas de intervención son válidos y fiables para la enseñanza del fútbol escolar, así como para comparar los efectos de ambas metodologías.

PALABRAS CLAVES: Validación, unidad didáctica, método, juez experto, V de Aiken.

\section{ABSTRACT}

The purpose of this study was to validate two intervention programs, corresponding to two didactic units, based in two different methodologies: Direct Instruction (DI) and Tactical Games Approach (TGA), for teaching school soccer. These two intervention programs were similar, with the same number of tasks, sessions, phases of play, specific contents and objectives. In the validation process participated 13 expert judges. Content validity was calculated by the coefficient of Aiken's $V$ and its confidence intervals. Also, internal consistency was calculated using Cronbach's $\alpha$. None of the 29 tasks of each intervention program was eliminated because they obtained values higher than the exact critical value $(V \geq .69)$. The internal consistency of the tasks, that formed both intervention programs together, was excellent $(\alpha=.97)$. For these reasons, both intervention programs are valid and reliable for teaching school soccer, as well as to compare the effects of these teaching methodologies.

KEY WORDS: Validation, soccer unit, method, expert judge, Aiken's V.

\section{INTRODUCCIÓN}

El método de enseñanza es el eje vertebrador sobre el que debe sustentarse la práctica deportiva (Kirk, 2005). Por ello, uno de los cometidos del profesor de Educación Física, a la hora de programar la enseñanza deportiva, es seleccionar el método que va a poner en práctica con el fin de que los estudiantes adquieran los aprendizajes lo más eficientemente posible. Destacan diferentes métodos de enseñanza del deporte: los centrados en el profesor (Teacher-Centered Approaches, TCAs) y los centrados en el alumno (Student-Centered Approaches, SCAs) (Zapatero, 2017). Blázquez (1999, p.255) diferencia ambos métodos según "el modo de implicar a los sujetos y el tipo y grado de aprendizaje que se desea".

En España, los métodos de enseñanza que más se han aplicado a lo largo del tiempo han sido los TCAs (Alarcón, Cárdenas, Miranda, Ureña, \& Piñar, 2010). Estos métodos se centran en la enseñanza de los gestos técnicos, incorporando la conciencia táctica una vez que dichos gestos se encuentran lo bastante desarrollados, de manera que la conciencia táctica no tiene lugar hasta el final 
del proceso de enseñanza (Abad, Benito, Giménez, \& Robles, 2013). Dentro de los TCAs, el más común es el método Direct Instruction (DI) (Metzler, 2011).

Posteriormente, con el fin de hacer frente a los inconvenientes de la concepción tecnicista y de mejorar el proceso de enseñanza-aprendizaje, surgen nuevas contribuciones de diferentes sectores de la Educación Física dando lugar a los SCAs (Abad et al., 2013). Los SCAs se basan en una enseñanza contextual fundamentada en el aprendizaje del qué, por qué y cuándo de los comportamientos técnico-tácticos a efectuar (Launder \& Piltz, 2006), es decir, tienen como fin la comprensión del juego a través de la conciencia táctica y de la apreciación del mismo (Gray \& Sproule, 2011). Dentro de los SCAs, el Tactical Games Approach (TGA) es el más común (Mitchell, Oslin, \& Griffin, 2013).

El posicionamiento metodológico del profesor va a determinar el diseño de las tareas motrices planteadas y su intervención en la práctica real (lbáñez, Feu, \& Cañadas, 2016). Ibáñez (2008) define las tareas de clase como aquellas herramientas empleadas por los profesores y entrenadores para desarrollar las habilidades y destrezas de los estudiantes. Las tareas diseñadas bajo los TCAs suelen ser inespecíficas y aisladas del contexto real del juego, o tareas específicas globales sin modificaciones cualitativas, en todo caso, cuantitativas variando el número de estudiantes (Alarcón et al., 2010). Por otro lado, los SCAs emplean juegos modificados y simplificados, o situaciones del juego real de competición, con o sin modificación de algún elemento del juego, de manera que los estudiantes analicen dichas situaciones para después, decidir la conducta técnico-táctica que consideren más adecuada y ejecutarla (Pérez-Muñoz, Yagüe, \& Sánchez-Sánchez, 2015; Serra, García-López, \& Sánchez-Mora, 2011).

El aspecto metodológico de la enseñanza deportiva es uno de los objetivos principales de estudio en el ámbito de la iniciación deportiva (Rodríguez, Mato, \& Pereira, 2016). Respecto a la mejora que produce cada método de enseñanza, diferentes autores afirman que no existen diferencias significativas entre los TCAs y los SCAs en cuanto a la enseñanza de gestos técnicos; sin embargo, en las variables relacionadas con la comprensión del juego como el conocimiento declarativo o la toma de decisiones, estos últimos alcanzan mejores resultados (Allison \& Thorpe, 1997; García y Ruiz, 2003; Turner \& Martinek, 1999). Por esta razón, las tendencias actuales proponen a los SCAs para la enseñanza de los deportes de invasión (García-Ceberino, Gamero, Feu, \& Ibáñez, 2020; González-Espinosa, Mancha-Triguero, García-Santos, Feu, e Ibáñez, 2019; Ibáñez et al., 2016). No obstante, los profesores de Educación Física siguen teniendo preferencia por los TCAs (Méndez, 2009). Dicha preferencia se debe a dos razones: la resistencia al cambio y la influencia ejercida por las instituciones deportivas nacionales (Devís \& Peiró, 1992).

De manera específica en el contexto escolar, existen diferentes estudios orientados al aspecto metodológico de la enseñanza del fútbol: para analizar los niveles de actividad estudiantil tras la enseñanza de unidades didácticas de fútbol centradas en el Game-Centred Approaches (GCAs) (Harvey, Song, Baek, \& Van der Mars, 2016); para examinar el impacto de una unidad de instrucción basada en el modelo híbrido Sport Education-Invasion Games Competence 
Model (IGCM) sobre el rendimiento y la comprensión del juego en el fútbol (Farias, Mesquita, \& Hastie, 2015); o para determinar los niveles de actividad física y la motivación autodeterminada tras la aplicación de unidades prolongadas (6-12 sesiones) mediante dos métodos pedagógicos: Direct Instruction y Tactical Games Model (TGM) (Smith et al., 2015).

La mayoría de los estudios centran su atención sobre los efectos de poner en práctica programas de intervención basados en la enseñanza técnica y táctica (Chatzopoulos, Tsormbatzoudis, \& Drakou, 2006; Gray \& Sproule, 2011; Mesquita, Farias, \& Hastie, 2012). Sin embargo, hasta donde se conoce, los estudios orientados al proceso de diseño (González-Espinosa, Ibáñez, \& Feu, 2017a) y validación, mediante jueces expertos, (González-Espinosa, Ibáñez, Feu, \& Galatti, 2017b) de los programas de intervención empleados son escasos. Es necesaria la difusión de estos estudios para ajustar y validar las tareas que conforman dichos programas a las características de las metodologías a estudiar (Feu, Ibáñez, García-Rubio, \& Antúnez, 2017). König \& Singrün (2013) destacan la importancia de garantizar la efectividad y sostenibilidad en la Educación Física mediante la validación empírica de las competencias técnicas y tácticas. La existencia y, posterior, aplicación de programas validados permitirá a los investigadores analizar el nivel de aprendizaje adquirido por los estudiantes tras su aplicación, así como contrastar los efectos de diversas metodologías en el aprendizaje de los deportes de invasión.

Por ello, este estudio tuvo como objetivo validar dos programas de intervención, correspondientes a dos unidades didácticas realizadas cada una en base a dos metodologías diferentes: Direct Instruction (DI) y Tactical Games Approach (TGA), para la enseñanza del fútbol escolar y que permitan su posterior implementación y comparación de los efectos producidos. Para tal validación, se analizó la validez de contenido y la consistencia interna de las tareas que conforman cada programa de intervención, todo ello a través de un juicio de expertos.

\section{MÉTODO}

\section{Diseño}

El presente estudio se encuadra dentro de los estudios instrumentales (Ato, López, \& Benavente, 2013), destinado a la validación de dos programas de intervención, unidades didácticas, que permitan obtener información válida y confiable (Corral, 2009) sobre el aprendizaje alcanzado por los estudiantes tras la enseñanza del fútbol en el contexto escolar.

\section{Participantes}

La muestra de participantes que colaboraron en este estudio se seleccionó de manera deliberada e intencional y de acuerdo con el grado en el que se ajustan a los criterios de inclusión establecidos por Rodríguez, Gil, \& García (1996). Así mismo, se buscó un panel de expertos con trayectoria reconocida en el tema de 
estudio y capaz de aportar juicios y valoraciones de ayuda al investigador (Escobar-Pérez \& Cuervo-Martínez, 2008).

Inicialmente, se solicitó la participación de 24 jueces expertos que cumplían los criterios de inclusión establecidos para formar parte de la muestra de jueces expertos que debían evaluar los programas diseñados. La muestra de participantes que finalmente cumplimentaron en tiempo y forma la información solicitada para validar ambos programas de intervención quedó conformada por un colectivo de 13 jueces expertos. El porcentaje de éxito en la participación fue de $54.17 \%$.

Los criterios de inclusión establecidos para formar parte de la muestra de jueces expertos fueron: i) Poseer el título de Doctor; ii) Ser profesor universitario en deportes de invasión y/o métodos de enseñanza del deporte (Pedagogía del Deporte); iii) Poseer la máxima titulación federativa (nivel III) en deportes de invasión; iv) Tener 10 años de experiencia como entrenador de deportes de invasión; v) Tener publicaciones sobre métodos de enseñanza del deporte y/o entrenamiento deportivo.

\section{Validez de contenido y consistencia interna de los programas de intervención}

\section{Validez de contenido}

La validez de contenido es definida como el grado en el que los ítems seleccionados representan adecuadamente al instrumento objeto de medición (Chacón-Moscoso et al., 2018). Para lograr un nivel óptimo de validez de contenido se empleó la técnica de valoración de jueces expertos (Cabero \& Barroso, 2013). Los jueces expertos valoraron los apartados «adecuación» y «elaboración» de cada una de las tareas que conforman ambos programas de intervención a través de una escala cuantitativa de tipo Likert de 1 a 10. Además, se requirió a los jueces expertos que realizaran valoraciones cualitativas sobre cada una de las tareas (García-Martín, Antúnez, \& Ibáñez, 2016).

- Grado de adecuación. Valoración cuantitativa emitida por cada experto para conocer la pertinencia de cada tarea al método de enseñanza, DI o TGA, en el que se incluye.

- Grado de elaboración. Cada experto valoró cuantitativamente la elaboración y redacción de las tareas que componen cada programa de intervención.

- Grado de valoración cualitativa. Se recogieron las propuestas emitidas por los jueces expertos para cada tarea con el fin de mejorarse.

\section{Consistencia interna}

La consistencia interna de las tareas que conforman cada programa de intervención fue medida mediante el coeficiente de $\alpha$ de Cronbach (Cronbach, 
1990). Este coeficiente es el método más empleado para el cálculo de la fiabilidad e indica hasta qué punto las respuestas a los ítems se encuentran relacionadas entre sí; por lo tanto, determinan que todos los ítems miden lo mismo y son sumables en una puntuación total única (Nunnally \& Bernstein, 1994).

\section{Instrumentos y materiales}

\section{Instrumentos}

Los dos programas de intervención, denominados Direct Instruction Soccer (DIS) y Tactical Games Approach Soccer (TGAS) (García-Ceberino, Feu, \& Ibáñez, 2019), persiguen la enseñanza del fútbol en el contexto escolar, así como comparar los métodos de enseñanza DI y TGA. En concreto, ambos programas de intervención están dirigidos a estudiantes de 5ํ y 6ํ de Educación Primaria.

Se diseñaron un total de 58 tareas, 29 tareas para cada programa de intervención. Cada tarea fue diseñada a través de las variables organizativas y pedagógicas establecidas por lbáñez et al. (2016): tiempo, organización y materiales, representación gráfica, breve descripción de la tarea, fase de juego, objetivo (ataque y/o defensa), medio de enseñanza, contenido específico y situación de juego. Estas variables se complementan con el apartado «feedback» para conocer la información proporcionada por el profesor a los estudiantes sobre la correcta ejecución de las tareas (Piéron, 1999). La comunicación profesor-estudiante debe ser acorde con la metodología aplicada; por lo tanto, se empleó un feedback descriptivo/prescriptivo para las tareas dirigidas al programa de intervención DIS y un feedback interrogativo para las tareas dirigidas al programa de intervención TGAS. En diversos estudios, el sistema de comunicación empleado en el aula se supone y no se refleja, a pesar de ser una variable que puede condicionar la propia tarea (Feu et al., 2017).

Ambos programas de intervención se distribuyen en 12 sesiones prácticas y presentan estructuras semejantes; por consiguiente, mismo número de tareas, sesiones, fases de juego, contenidos específicos y objetivos (ataque y/o defensa) (García-Ceberino et al., 2019). El número de sesiones fue establecido de acuerdo a López \& Castejón (2005), quienes afirman que este tipo de estudios necesitan aplicarse en periodos largos de tiempo con el fin de obtener más datos y más fiables. Las sesiones no presentan la estructura clásica (animación-parte fundamental-vuelta a la calma) de una sesión de Educación Física (SáenzLópez, 1997). Cada sesión consta de un total de 4 tareas con una duración de 10 minutos cada tarea. Las sesiones se estructuran de forma progresiva, desde las tareas más simples (actividades de activación) hasta las tareas más complejas (actividades de culminación) (Ibáñez, 2009). González-Espinosa et al. (2017a) diseñaron dos programas de intervención semejantes a los presentados en este estudio, destinados a la enseñanza del baloncesto. 


\section{Materiales}

Los datos fueron recopilados a través del paquete ofimático Microsoft Excel 2013. Para el cálculo del coeficiente de $V$ de Aiken y sus intervalos de confianza se empleó el programa gratuito Visual Basic 6.0, diseñado por Merino \& Livia (2009). Por último, se utilizó el programa estadístico SPSS 21.0 (IBM Corp. Released 2012. IBM SPSS Statistics for Windows, Version 21. Armonk, NY: IBM Corp) para el análisis de consistencia interna de las tareas que conforman cada programa de intervención de manera independiente y conjunta.

\section{Procedimiento}

Para el desarrollo de este estudio se siguieron una serie de acciones ordenadas cronológicamente. En primer lugar, se realizó una revisión de la literatura existente sobre el objeto de estudio a partir de la cual se diseñó un primer borrador de los dos programas de intervención (unidades didácticas). Seguidamente, se establecieron los criterios de inclusión que debían cumplir los sujetos que conforman el panel de expertos. Una vez seleccionada una muestra de jueces expertos, se les envió por correo electrónico la documentación necesaria para validar ambos programas de intervención. Entre los documentos se incluía una presentación formal e institucional del estudio, el desarrollo de las tareas correspondientes a ambos programas de intervención y una hoja de evaluación donde los jueces expertos tenían que valorar cuantitativamente el grado de adecuación y elaboración de cada una de las tareas, así como realizar valoraciones cualitativas que permitieran la mejora de las mismas. En dichos documentos se informaba a los jueces expertos de la edad de los destinatarios, de la distribución de las tareas en las distintas sesiones y del orden que ocupaban en las mismas, así como del sistema de comunicación.

Posteriormente, se procedió a la recogida de las valoraciones aportadas por el panel de expertos y al análisis de las mismas, siendo necesario realizar dos rondas de evaluación. Tras analizar los resultados obtenidos en la primera ronda de evaluación, se mantuvieron las tareas que alcanzaron una puntuación óptima, se mejoraron las tareas que los resultados así lo demandaban según las sugerencias aportadas por los jueces expertos y por último, se reelaboraron aquellas tareas que no obtuvieron una puntuación adecuada tras el cálculo de validez de contenido (Ortega, Jiménez, Palao, \& Sainz, 2008). Estas últimas fueron enviadas al panel de expertos para ser valoradas nuevamente de forma cuantitativa y cualitativa en una segunda ronda de evaluación.

Finalmente, los dos programas de intervención, DIS y TGAS, quedaron definidos.

\section{Análisis de datos}

La validación de los programas de intervención a partir de las valoraciones de los jueces expertos se calculó mediante el índice de validez de contenido, denominado coeficiente de $V$ de Aiken (Aiken, 1985). Dicho coeficiente permite cuantificar la relevancia de un ítem de acuerdo a la opinión de un grupo de jueces 
expertos. Para el cálculo del coeficiente de $V$ de Aiken se empleó la ecuación algebraica modificada por Penfield \& Giacobbi (2004).

$$
V=\frac{\bar{X}-I}{k}
$$

Para su cálculo, se utilizó el programa gratuito Visual Basic 6.0 (Merino \& Livia, 2009). Este programa posibilita obtener tres factores: el rango de las valoraciones (valoración máxima - valoración mínima), el coeficiente de $V$ de Aiken y los intervalos de confianza en los niveles de 90\%, 95\% y 99\% mediante el método score (Penfield \& Giacobbi, 2004).

El valor crítico exacto de aceptación de la $V$ de Aiken se calculó mediante la fórmula inicial propuesta por Aiken (1985), aplicando el teorema del límite central para grandes muestras $(m>25)$. El número de jueces fue $13(n)$, el de ítems 58 $(m)$, con un rango de respuesta de $10(c)$; aplicándose el 95\% o 99\% de nivel de confianza $(z)$.

$$
\mathrm{V}=\frac{\mathrm{z}}{0.2 \sqrt{\frac{3 \mathrm{mn}(\mathrm{c}-1)}{(\mathrm{c}+1)}}}+0.5
$$

Para lograr el valor crítico exacto se consideró el nivel de confianza de $95 \%$, obteniéndose un valor de .69. Asimismo, para obtener el punto de corte de modificación de las tareas se consideró el nivel de confianza de 99\%, obteniéndose un valor de .77.

Por lo tanto, se eliminarán las tareas con valores inferiores con un $95 \%$ de confianza $(V<.69)$, se modificaran las tareas con valores comprendidos entre un $95 \%$ y $99 \%$ de confianza $(V=.69$ y .77$)$ y se consideraran como óptimas las tareas con valores superiores con un $99 \%$ de confianza $(V>.77)$ (Tabla 1).

Tabla 1. Criterios a seguir para la aceptación, modificación o eliminación de las tareas

\begin{tabular}{ccccc}
\hline & & \multicolumn{3}{c}{ Elaboración } \\
\cline { 3 - 5 } & $>.77$ & $>.77$ & {$[.69-.77]$} & $<.69$ \\
\multirow{3}{*}{ Adecuación } & Correcta & Se modifica Elaboración & Se modifica Elaboración \\
& {$[.69-.77]$} & Se modifica Adecuación & Se modifica A+E & Se modifica A+E \\
& $<.69$ & Se elimina & Se elimina & Se elimina \\
\hline
\end{tabular}

El análisis de consistencia interna de las tareas que constituyen ambos programas de intervención se calculó mediante el coeficiente de $\alpha$ de Cronbach (Cronbach, 1990; Field, 2013).

\section{RESULTADOS}

En las Tablas 2 y 3 se exponen los resultados obtenidos tras el cálculo del coeficiente de $V$ de Aiken y sus intervalos de confianza al $95 \%$ y $99 \%$ de las 
tareas que conforman los programas de intervención DIS y TGAS, respectivamente.

Tabla 2. Resultados del coeficiente de $V$ de Aiken e IC de las tareas del programa de intervención DIS

\begin{tabular}{|c|c|c|c|c|c|c|c|c|c|c|c|c|}
\hline \multirow[b]{3}{*}{ Tarea } & \multicolumn{6}{|c|}{ Adecuación } & \multicolumn{6}{|c|}{ Elaboración } \\
\hline & \multirow[b]{2}{*}{$M \pm D T$} & \multirow[b]{2}{*}{$V$} & \multicolumn{2}{|c|}{$95 \%$ IC } & \multicolumn{2}{|c|}{$99 \%$ IC } & \multirow[b]{2}{*}{$M \pm D T$} & \multirow[b]{2}{*}{ V } & \multicolumn{2}{|c|}{$95 \%$ IC } & \multicolumn{2}{|c|}{$99 \%$ IC } \\
\hline & & & $\operatorname{lnf}$ & Sup & $\operatorname{lnf}$ & Sup & & & $\operatorname{lnf}$ & Sup & $\operatorname{lnf}$ & $\overline{\text { Sup }}$ \\
\hline 1 & $9.92 \pm .28$ & .99 & .95 & 1.00 & .93 & 1.00 & $8.85 \pm 2.27$ & .87 & .80 & .92 & .77 & .93 \\
\hline 2 & $8.77 \pm 2.31$ & .86 & .79 & .91 & .76 & .92 & $8.15 \pm 2.51$ & .79 & .71 & .86 & .68 & .87 \\
\hline 3 & $9.92 \pm .28$ & .99 & .95 & 1.00 & .93 & 1.00 & $8.77 \pm 2.24$ & .86 & .79 & .91 & .76 & .92 \\
\hline 4 & $7.77 \pm 2.01$ & $.75^{\star}$ & .67 & .82 & .64 & .84 & $8.15 \pm 1.95$ & .79 & .71 & .86 & .68 & .87 \\
\hline 5 & $9.92 \pm .28$ & .99 & .95 & 1.00 & .93 & 1.00 & $8.77 \pm 2.13$ & .86 & .79 & .91 & .76 & .92 \\
\hline 6 & $9.77 \pm .44$ & .97 & .93 & .99 & .90 & .99 & $8.46 \pm 2.60$ & .83 & .75 & .89 & .72 & .90 \\
\hline 7 & $9.54 \pm .97$ & .95 & .89 & .98 & .87 & .98 & $8.62 \pm 2.06$ & .85 & .77 & .90 & .74 & .91 \\
\hline 8 & $9.31 \pm .95$ & .92 & .86 & .96 & .84 & .97 & $9.00 \pm 1.08$ & .89 & .82 & .93 & .79 & .94 \\
\hline 9 & $9.92 \pm .28$ & .99 & .95 & 1.00 & .93 & 1.00 & $8.77 \pm 2.24$ & .86 & .79 & .91 & .76 & .92 \\
\hline 10 & $9.62 \pm .77$ & .96 & .90 & .98 & .88 & .98 & $8.46 \pm 2.57$ & .83 & .75 & .89 & .72 & .90 \\
\hline 11 & $9.77 \pm .60$ & .97 & .93 & .99 & .90 & .99 & $8.54 \pm 2.37$ & .84 & .76 & .89 & .73 & .91 \\
\hline 12 & $9.46 \pm .78$ & .94 & .88 & .97 & .86 & .98 & $9.00 \pm 1.00$ & .89 & .82 & .93 & .79 & .94 \\
\hline 13 & $8.38 \pm 1.85$ & .82 & .74 & .88 & .71 & .89 & $8.08 \pm 2.75$ & .79 & .70 & .85 & .68 & .87 \\
\hline 14 & $9.54 \pm .88$ & .95 & .89 & .98 & .87 & .98 & $8.23 \pm 2.39$ & .80 & .72 & .86 & .69 & .88 \\
\hline 15 & $9.69 \pm .85$ & .96 & .91 & .99 & .89 & .99 & $8.54 \pm 1.76$ & .84 & .76 & .89 & .73 & .90 \\
\hline 16 & $9.69 \pm .63$ & .96 & .91 & .99 & .89 & .99 & $8.46 \pm 1.90$ & .83 & .75 & .89 & .72 & .90 \\
\hline 17 & $9.69 \pm .63$ & .96 & .91 & .99 & .89 & .99 & $8.69 \pm 1.70$ & .85 & .78 & .91 & .75 & .92 \\
\hline 18 & $9.77 \pm .60$ & .97 & .93 & .99 & .90 & .99 & $8.62 \pm 1.71$ & .85 & .77 & .90 & .74 & .91 \\
\hline 19 & $9.77 \pm .83$ & .97 & .93 & .99 & .90 & .99 & $8.31 \pm 2.46$ & .81 & .73 & .87 & .70 & .89 \\
\hline 20 & $8.38 \pm 1.76$ & .82 & .74 & .88 & .71 & .89 & $8.23 \pm 1.59$ & .80 & .72 & .86 & .69 & .88 \\
\hline 21 & $7.54 \pm 2.99$ & $.73^{*}$ & .64 & .80 & .61 & .82 & $7.54 \pm 2.93$ & $.73^{\star}$ & .64 & .80 & .61 & .82 \\
\hline 22 & $9.23 \pm 1.59$ & .91 & .85 & .95 & .82 & .96 & $7.38 \pm 3.23$ & $.71^{*}$ & .62 & .78 & .59 & .80 \\
\hline 23 & $8.54 \pm 1.94$ & .84 & .76 & .89 & .73 & .91 & $7.85 \pm 2.70$ & $.76^{*}$ & .68 & .83 & .65 & .85 \\
\hline 24 & $8.85 \pm 1.52$ & .87 & .80 & .92 & .77 & .93 & $8.46 \pm 2.26$ & .83 & .75 & .89 & .72 & .90 \\
\hline 25 & $8.77 \pm 1.48$ & .86 & .79 & .91 & .76 & .92 & $8.77 \pm 1.30$ & .86 & .79 & .91 & .76 & .92 \\
\hline 26 & $8.23 \pm 1.59$ & .80 & .72 & .86 & .69 & .88 & $8.62 \pm 1.45$ & .85 & .77 & .90 & .74 & .91 \\
\hline 27 & $8.77 \pm 1.24$ & .86 & .79 & .91 & .76 & .92 & $8.62 \pm 1.19$ & .85 & .77 & .90 & .74 & .91 \\
\hline 28 & $8.23 \pm 1.79$ & .80 & .72 & .86 & .69 & .88 & $8.46 \pm 1.56$ & .83 & .75 & .89 & .72 & .90 \\
\hline 29 & $7.23 \pm 2.65$ & $.69^{*}$ & .60 & .77 & .57 & .79 & $8.62 \pm 1.50$ & .85 & .77 & .90 & .74 & .91 \\
\hline
\end{tabular}

Nota: $M=$ Promedio; $D T=$ Desviación típica; $V=$ Valor de $V$ de Aiken; $I C=$ Intervalo de confianza; Inf = Límite inferior; Sup = Límite superior 
Rev.int.med.cienc.act.fís.deporte - vol. 20 - número 78 - ISSN: 1577-0354

Tabla 3. Resultados del coeficiente de $V$ de Aiken e IC de las tareas del programa de intervención TGAS

\begin{tabular}{|c|c|c|c|c|c|c|c|c|c|c|c|c|}
\hline \multirow[b]{3}{*}{ Tarea } & \multicolumn{6}{|c|}{ Adecuación } & \multicolumn{6}{|c|}{ Elaboración } \\
\hline & \multirow[b]{2}{*}{$M \pm D T$} & \multirow[b]{2}{*}{$V$} & \multicolumn{2}{|c|}{$95 \%$ IC } & \multicolumn{2}{|c|}{$99 \%$ IC } & \multirow[b]{2}{*}{$M \pm D T$} & \multirow[b]{2}{*}{$V$} & \multicolumn{2}{|c|}{$95 \%$ IC } & \multicolumn{2}{|c|}{$99 \%$ IC } \\
\hline & & & $\operatorname{lnf}$ & Sup & $\operatorname{lnf}$ & Sup & & & Inf & Sup & $\operatorname{lnf}$ & Sup \\
\hline 1 & $8.54 \pm 1.39$ & .84 & .76 & .89 & .73 & .91 & $8.85 \pm 1.46$ & .87 & .80 & .92 & .77 & .93 \\
\hline 2 & $8.69 \pm 1.84$ & .85 & .78 & .91 & .75 & .92 & $8.23 \pm 2.01$ & .80 & .72 & .86 & .69 & .88 \\
\hline 3 & $8.77 \pm 1.83$ & .86 & .79 & .91 & .76 & .92 & $8.46 \pm 1.94$ & .83 & .75 & .89 & .72 & .90 \\
\hline 4 & $9.31 \pm 1.03$ & .92 & .86 & .96 & .84 & .97 & $8.46 \pm 1.39$ & .83 & .75 & .89 & .72 & .90 \\
\hline 5 & $8.46 \pm 2.54$ & .83 & .75 & .89 & .72 & .90 & $7.85 \pm 2.54$ & $.76^{*}$ & .68 & .83 & .65 & .85 \\
\hline 6 & $7.31 \pm 2.29$ & $.70^{*}$ & .61 & .78 & .58 & .80 & $7.85 \pm 2.27$ & $.76^{*}$ & .68 & .83 & .65 & .85 \\
\hline 7 & $7.85 \pm 2.61$ & $.76^{*}$ & .68 & .83 & .65 & .85 & $7.77 \pm 2.39$ & $.75^{*}$ & .67 & .82 & .64 & .84 \\
\hline 8 & $8.62 \pm 1.71$ & .85 & .77 & .90 & .74 & .91 & $8.08 \pm 1.98$ & .79 & .70 & .85 & .68 & .87 \\
\hline 9 & $8.92 \pm 1.44$ & .88 & .81 & .93 & .78 & .94 & $8.15 \pm .1 .63$ & .79 & .71 & .86 & .68 & .87 \\
\hline 10 & $8.77 \pm 2.45$ & .86 & .79 & .91 & .76 & .92 & $8.92 \pm 1.32$ & .88 & .81 & .93 & .78 & .94 \\
\hline 11 & $8.31 \pm 1.70$ & .81 & .73 & .87 & .70 & .89 & $8.69 \pm 1.49$ & .85 & .78 & .91 & .75 & .92 \\
\hline 12 & $7.46 \pm 2.18$ & $.72^{*}$ & .63 & .79 & .60 & .81 & $7.38 \pm 2.26$ & $.71^{*}$ & .62 & .78 & .59 & .80 \\
\hline 13 & $8.00 \pm 1.53$ & .78 & .69 & .84 & .67 & .86 & $8.00 \pm 1.63$ & .78 & .69 & .84 & .67 & .86 \\
\hline 14 & $8.08 \pm 1.61$ & .79 & .70 & .85 & .68 & .87 & $8.00 \pm 1.63$ & .78 & .69 & .84 & .67 & .86 \\
\hline 15 & $8.00 \pm 1.53$ & .78 & .69 & .84 & .67 & .86 & $8.00 \pm 1.63$ & .78 & .69 & .84 & .67 & .86 \\
\hline 16 & $7.92 \pm 1.61$ & $.77^{*}$ & .68 & .83 & .66 & .8 & $8.08 \pm 1.71$ & .79 & .70 & .85 & .68 & .87 \\
\hline 17 & $8.92 \pm 1.32$ & .88 & .81 & .93 & .78 & .94 & $8.38 \pm 2.18$ & .82 & .74 & .88 & .71 & .89 \\
\hline 18 & $7.54 \pm 2.30$ & $.73^{*}$ & .64 & .80 & .61 & .82 & $7.77 \pm 2.20$ & $.75^{*}$ & .67 & .82 & .64 & .84 \\
\hline 19 & $7.62 \pm 1.80$ & $.73^{\star}$ & .65 & .81 & .62 & .82 & $7.85 \pm 1.41$ & $.76^{*}$ & .68 & .83 & .65 & .85 \\
\hline 20 & $8.38 \pm 2.18$ & .82 & .74 & .88 & .71 & .89 & $8.85 \pm 1.77$ & .87 & .80 & .92 & .77 & .93 \\
\hline 21 & $7.46 \pm 1.76$ & $.72^{*}$ & .63 & .79 & .60 & .81 & $7.69 \pm 1.49$ & $.74^{*}$ & .66 & .81 & .63 & .83 \\
\hline 22 & $7.85 \pm 1.77$ & $.76^{*}$ & .68 & .83 & .65 & .85 & $7.85 \pm 1.82$ & $.76^{*}$ & .68 & .83 & .65 & .85 \\
\hline 23 & $8.69 \pm 1.55$ & .85 & .78 & .91 & .75 & .92 & $7.92 \pm 1.80$ & $.77^{*}$ & .68 & .83 & .66 & .85 \\
\hline 24 & $8.69 \pm .85$ & .85 & .78 & .91 & .75 & .92 & $8.08 \pm 1.19$ & .79 & .70 & .85 & .68 & .87 \\
\hline 25 & $8.69 \pm 1.38$ & .85 & .78 & .91 & .75 & .92 & $8.38 \pm 1.76$ & .82 & .74 & .88 & .71 & .89 \\
\hline 26 & $8.38 \pm 1.56$ & .82 & .74 & .88 & .71 & .89 & $8.08 \pm 1.85$ & .79 & .70 & .85 & .68 & .87 \\
\hline 27 & $9.15 \pm 1.21$ & .90 & .84 & .95 & .81 & .95 & $8.46 \pm 1.71$ & .83 & .75 & .89 & .72 & .90 \\
\hline 28 & $8.92 \pm 1.50$ & .88 & .81 & .93 & .78 & .94 & $8.46 \pm 1.76$ & .83 & .75 & .89 & .72 & .90 \\
\hline 29 & $9.00 \pm 1.47$ & .89 & .82 & .93 & .79 & .94 & $8.38 \pm 1.71$ & .82 & .74 & .88 & .71 & .89 \\
\hline
\end{tabular}

Nota: $M=$ Promedio; $D T=$ Desviación típica; $V=$ Valor de $V$ de Aiken; $I C=$ Intervalo de confianza; Inf = Límite inferior; Sup = Límite superior

En la Tabla 4 se detallan, a modo de ejemplo, algunas de las valoraciones cualitativas emitidas por los jueces expertos para la mejora de las tareas de ambos programas de intervención. 
Tabla 4. Valoraciones cualitativas emitidas por los jueces expertos

\begin{tabular}{|c|c|c|}
\hline Tarea & Valoración cualitativa & Acción \\
\hline 4 (DIS) & $\begin{array}{c}\text { J3: "Juego con incertidumbre y toma de } \\
\text { decisiones" }\end{array}$ & $\begin{array}{l}\text { Se han establecido reglas y fijado funciones a } \\
\text { los niños para suprimir la toma de decisiones }\end{array}$ \\
\hline 5 (DIS) & J11: "Reducir las esperas" & Se ha aumentado el número de filas \\
\hline 7 (DIS) & J2: “Establecer el ritmo de ejecución” & $\begin{array}{c}\text { Se han indicado los momentos de intervención } \\
\text { de los niños, los cuales serán marcados por el } \\
\text { profesor }\end{array}$ \\
\hline 26 (DIS) & $\begin{array}{c}\text { J8: "Indicar la distancia del espacio } \\
\text { delimitado" }\end{array}$ & $\begin{array}{c}\text { Se ha indicado la dimensión del espacio de } \\
\text { juego: } 7 \times 7 \mathrm{mts}\end{array}$ \\
\hline 27 (DIS) & J1: "Los defensores salgan detrás" & $\begin{array}{c}\text { Se ha retrasado la posición de partida inicial } \\
\text { de los defensores con respecto a la de los } \\
\text { atacantes }\end{array}$ \\
\hline 2 (TGAS) & $\begin{array}{l}\text { J1: "Facilitar el ataque con } \\
\text { superioridades numéricas" }\end{array}$ & $\begin{array}{l}\text { Se ha añadido un niño comodín, quien juega } \\
\text { con el equipo que esté en posesión del balón }\end{array}$ \\
\hline 8 (TGAS) & $\begin{array}{l}\text { J2: "Buscar una situación más real de } \\
\text { juego" }\end{array}$ & $\begin{array}{c}\text { Se ha adaptado la tarea a una situación más } \\
\text { real de juego }\end{array}$ \\
\hline 12 (TGAS) & J1: "Demasiado tiempo de espera" & $\begin{array}{l}\text { Se han incluido varias situaciones de } 1 \times 1 \text { a la } \\
\text { vez }\end{array}$ \\
\hline 19 (TGAS) & J8: "Indicar medida del espacio" & $\begin{array}{l}\text { Se ha indicado la dimensión del espacio de } \\
\text { juego: } 9 \times 9 \mathrm{mts}\end{array}$ \\
\hline 29 (TGAS) & $\begin{array}{l}\text { J9: "Jugar con dos porterías pequeñas } \\
\text { en cada fondo del campo" }\end{array}$ & $\begin{array}{c}\text { Se ha añadido otra portería pequeña para } \\
\text { poder jugar con dos porterías, una en cada } \\
\text { fondo del campo }\end{array}$ \\
\hline
\end{tabular}

Nota: J=Juez experto

Por último, en la Tabla 5 se exponen los resultados de consistencia interna de las tareas que conforman los programas de intervención DIS y TGAS de manera independiente, así como la consistencia interna del total de las tareas de ambos programas de intervención, tras el cálculo del coeficiente de $\alpha$ de Cronbach.

Tabla 5. Consistencia interna de las tareas que conforman los programas de intervención DIS y TGAS

\begin{tabular}{|c|c|c|c|c|c|c|c|c|c|}
\hline \multirow[b]{3}{*}{$\alpha$ Cronbach } & \multicolumn{3}{|c|}{ DIS } & \multicolumn{3}{|c|}{ TGAS } & \multicolumn{3}{|c|}{ DIS y TGAS } \\
\hline & $A$ & $E$ & Total & $A$ & $E$ & Total & $A$ & $E$ & Total \\
\hline & .87 & .96 & .96 & .93 & .95 & .97 & .93 & .96 & .97 \\
\hline Válidos & 13 & 13 & 13 & 13 & 13 & 13 & 13 & 13 & 13 \\
\hline$N$ & 13 & 13 & 13 & 13 & 13 & 13 & 13 & 13 & 13 \\
\hline
\end{tabular}

\section{DISCUSIÓN}

Este estudio tuvo por objetivo la validación de dos programas de intervención, unidades didácticas, realizados sobre la base de dos metodologías de enseñanza diferentes: DI y TGA; alcanzando ambos programas niveles óptimos de validez y consistencia interna. Para ello, se han seguido los procedimientos metodológicos recomendados en la literatura científica (Anguera \& HernándezMendo, 2013; Bulger \& Housner, 2007; Escobar-Pérez \& Cuervo-Martínez, 2008), así como estudios similares (Ibáñez, Martínez-Fernández, GonzálezEspinosa, García-Rubio, \& Feu, 2019; Torres-Luque, Fernández-García, Cabello-Manrique, Giménez-Egido, \& Ortega-Toro, 2018; Villarejo, Ortega, Gómez, \& Palao, 2014).

Para la validación de los programas de intervención se empleó el panel de expertos, una de las técnicas más utilizadas en la investigación educativa para 
la evaluación de instrumentos (Cabero \& Barroso, 2013). Este estudio cuenta con un colectivo de 13 jueces expertos, número adecuado para ofrecer una estimación aceptable para la validez de contenido de las tareas que constituyen ambos programas de intervención. Barahona (2004); Jiménez, Salazar, \& Morera (2013); Robles, Robles, Giménez, \& Abad (2016) señalan que se necesitan un mínimo de diez sujetos para ofrecer una estimación aceptable para la validez de contenido de un instrumento de validación. El número de jueces expertos que colaboraron en este estudio es superior al mínimo establecido en la literatura científica, contando con un porcentaje de éxito del $54,17 \%$. Para la selección de los jueces expertos se establecieron una serie de criterios de inclusión (Rodríguez et al., 1996). Estudios para la validación de diferentes instrumentos también fijaron criterios de inclusión para la selección de jueces expertos: para validar acciones básicas durante el lanzamiento de personas ciegas a portería en situaciones de juego y durante el penalti (Gamonales, León, Muñoz, González-Espinosa, \& Ibáñez, 2018); para validar una hoja de observación que permita conocer la actuación arbitral durante partidos de baloncesto (García-Santos \& Ibáñez, 2016); o para validar cuestionarios para analizar el proceso de formación en jugadores expertos de deportes colectivos (García-Martín et al., 2016).

La intervención de los jueces expertos se dirigió hacia la valoración de la adecuación y elaboración de los ítems/tareas de los programas de intervención. Este procedimiento es el habitual en estudios que desarrollan instrumentos como cuestionarios para valorar las preferencias y satisfacciones en jóvenes jugadores de baloncesto (Ortega et al., 2008); o analizar las acciones tácticas en rugby (Villarejo et al., 2014). De manera específica, existen validaciones de programas de intervención para la enseñanza del baloncesto en el contexto escolar (González-Espinosa et al., 2017b).

La validez de contenido de las tareas que conforman ambos programas de intervención se calculó mediante el coeficiente de $V$ de Aiken. Para ello, se aplicó la ecuación algebraica modificada por Penfield \& Giacobbi (2004). Esta fórmula ha sido utilizada en diferentes estudios para la validación de nuevos instrumentos dirigidos al ámbito deportivo (Almonacid-Fierro, Feu, \& Vizuete, 2018; Collet, Nascimento, Folle, \& Ibáñez, 2018; Ibáñez et al., 2019). El valor crítico exacto de aceptación de las tareas se calculó mediante la fórmula inicial propuesta por Aiken (1985), aplicando el teorema del límite central para grandes muestras. Igual que los estudios citados anteriormente, para el cálculo del valor crítico exacto se consideró el nivel de confianza de $95 \%$. Asimismo, para obtener el punto de corte de modificación de las tareas se consideró el nivel de confianza de $99 \%$. En este estudio, el nivel de exigencia para la aceptación o eliminación de un ítem/tarea es muy alto. Hasta donde se conoce, se trata del primer estudio que emplea el $99 \%$ de confianza para determinar la aceptación y modificación de un ítem/tarea en el proceso de validación. Para aceptar la validez de un instrumento en estudios iniciales se puede optar por el valor crítico liberal .50 (Aiken, 1985), o por el valor crítico conservador .70 (Charter, 2003). El valor crítico empleado en este estudio está condicionado por el número de ítems/tareas, el número de jueces expertos y el rango de respuesta. A partir de este valor, se establece un rango para la modificación de un ítem/tarea (entre $95 \%$ y $99 \%$ de confianza), o para la aceptación absoluta de un ítem/tarea ( $V>$ 
99\% de confianza). A pesar de ello, se han aceptado todas las sugerencias de los jueces expertos con el objetivo de mejorar los programas de intervención.

Los resultados indican que ninguna de las 29 tareas que componen cada programa de intervención debe ser eliminada al poseer valores en el coeficiente de $V$ de Aiken superiores al valor crítico exacto. Solo cinco tareas, las tareas 4, $21,22,23$ y 29, que constituyen el programa de intervención DIS fueron modificadas al obtener valores comprendidos en el intervalo [.69 -.77] en los apartados «adecuación» y/o «elaboración». De igual modo, las tareas 5, 6, 7, $12,16,18,19,21,22$ y 23 que conforman el programa de intervención TGAS también fueron modificadas al presentar valores comprendidos en dicho intervalo en los apartados «adecuación» y/o «elaboración». Para efectuar tales modificaciones, se siguieron las valoraciones cualitativas emitidas por los jueces expertos (Bulger \& Housner, 2007; Ortega, Calderón, Palao, \& Puigcerver, 2009; Ortega-Toro, García-Angulo, Giménez-Egido, García-Angulo, \& Palao, 2019). Las propuestas de mejora emitidas por los jueces expertos para la mayoría de las tareas se dirigen hacia las dimensiones de los espacios de juego, número de estudiantes, ritmos de ejecución, etc. El control de los aspectos formales que definen una tarea es básico para el diseño de tareas basadas en los SCAs.

La consistencia interna fue calculada a través del coeficiente de $\alpha$ de Cronbach (Cronbach, 1990). Se calculó la consistencia interna de las tareas que constituyen cada programa de intervención de manera independiente, así como de las tareas de ambos programas de intervención de manera conjunta. Las tareas que conforman el programa de intervención DIS obtuvieron un valor de .96 y las tareas que constituyen el programa de intervención TGAS alcanzaron un valor de .97. Por último, el total de tareas de ambos programas de intervención obtuvo un valor de .97. Cuando las investigaciones persiguen la fiabilidad de instrumentos mediante comparación de grupos, puede aceptarse un valor del coeficiente de $\alpha$ de Cronbach cercano a .70 (Nunnally, 1978); no obstante, es recomendable obtener valores en este coeficiente mayores a .80 (Polit \& Hungler, 2000), considerándose como excelentes los valores superiores a .90 (George \& Mallery, 2003). Según Field (2013), para interpretar los datos y que un instrumento sea fiable, cuanto más se acerquen los datos al extremo del índice 1.00; mejor será la fiabilidad de un instrumento. En este estudio la fiabilidad de los programas de intervención es excelente.

Por último, entre las limitaciones encontradas en este estudio destacar el escaso número de programas de intervención destinados a comparar los métodos de enseñanza deportiva en el contexto escolar (González-Espinosa et al., 2017a; González-Espinosa et al., 2017b). Además, señalar la dificultad para lograr el mínimo de jueces expertos requeridos, diez (Barahona, 2004; Jiménez et al., 2013; Robles et al., 2016).

\section{CONCLUSIONES}

Las tareas que componen los programas de intervención DIS y TGAS presentan niveles óptimos de validez y consistencia interna; por lo tanto, ambos programas son considerados válidos y fiables para la enseñanza del fútbol en el contexto escolar por parte de los profesores de Educación Física. 
En este estudio se ha empleado como referencias para la aceptación, modificación y eliminación de los ítems/tareas, los valores resultantes de la aplicación en el coeficiente de $V$ de Aiken de los intervalos de confianza al 95\% y $99 \%$.

La validación de este tipo de programas de intervención permitirá a los investigadores analizar el nivel de aprendizaje alcanzado por los estudiantes tras su puesta en práctica, así como comparar los efectos de los métodos de enseñanza DI y TGA.

\section{REFERENCIAS}

Abad, M. T., Benito, P. J., Giménez, F. J., \& Robles, J. (2013). Fundamentos pedagógicos de la enseñanza comprensiva del deporte: una revisión de la literatura. Cultura, Ciencia y Deporte, 23(8), 137-146. doi:10.12800/ccd.v8i23.300

Aiken, L. R. (1985). Three coefficients for analyzing the reliability and validity of ratings. Educational and Psychological Measurement, 45(1), 131-142. doi:10.1177/0013164485451012

Alarcón, F., Cárdenas, D., Miranda, M. T., Ureña, N., \& Piñar, M. I. (2010). La metodología de enseñanza en los deportes de equipo. Revista de Investigación en Educación, 7, 91-103.

Allison, S., \& Thorpe, R. (1997). A comparison of the effectiveness of two approaches to teaching games within physical education. A skills approach versus a games for understanding approach. British Journal of Physical Education, 28(3), 9-13.

Almonacid-Fierro, A., Feu, S., \& Vizuete, M. (2018). Validación de un cuestionario para medir el Conocimiento Didáctico del Contenido en el profesorado de Educación Física. Retos. Nuevas Tendencias en Educación Física, Deporte y Recreación, 37, 132-137.

Anguera, M. T., \& Hernández-Mendo, A. H. (2013). Metodología observacional en el ámbito del deporte. E-balonmano.com: Revista de Ciencias del Deporte, 9(3), 135-160.

Ato, M., López, J. J., \& Benavente, A. (2013). Un sistema de clasificación de los diseños de investigación en psicología. Anales de Psicología, 29(3), 10381059. doi:10.6018/analesps.29.3.178511

Barahona, E. (2004). Estudio de validez del Cuestionario de Prácticas Pedagógicas para la Creatividad (CPPC). Psykhe, 13(1), 157-174. doi:10.4067/S0718-22282004000100013

Blázquez, D. (1995). La iniciación deportiva y el deporte escolar. Barcelona: Inde.

Bulger, S. M., \& Housner, L. D. (2007). Modified Delphi investigation of exercise science in physical education teacher education. Journal of Teaching in Physical Education, 26(1), 57-80. doi:10.1123/jtpe.26.1.57

Cabero, J., \& Barroso, J. (2013). La utilización del juicio de experto para la evaluación de TIC: el coeficiente de competencia experta. Bordón, 65(2), 25-38. doi:10.13042/brp.2013.65202

Chacón-Moscoso, S., Sanduvete-Chaves, S., Anguera, M. T., Losada, J. L., Portell, M., \& Lozano-Lozano, J. A. (2018). Preliminary checklist for 
reporting observational studies in sports areas: content validity. Frontiers in Psychology, 9, 291. doi:10.3389/fpsyg.2018.00291

Charter, R. A. (2003). A breakdown of reliability coefficients by test type and reliability method, and the clinical implications of low reliability. Journal of General Psychology, 130(3), 290-304. doi:10.1080/00221300309601160

Chatzopoulos, D., Tsormbatzoudis, H., \& Drakou, A. (2006). Combinations of technique and games approaches: Effects on game performance and motivation. Journal of Human Movement Studies, 50(3), 157-170.

Collet, C., Nascimento, J. V., Folle, A., \& Ibáñez, S. J. (2018). Construcción y validación de un instrumento para el análisis de la formación deportiva en voleibol. Cuadernos de Psicología del Deporte, 19(1), 76-89. doi:10.6018/cpd.326361

Corral, Y. (2009). Validez y confiabilidad de los instrumentos de investigación para la recolección de datos. Revista de Ciencias de la Educación, 19(33), 228-247.

Cronbach, L. J. (1990). Essentials of psychological testing (5 $5^{\text {th }}$ Ed.). New York: Harper \& Row.

Devís, J., \& Peiró, C. (1992). Nuevas perspectivas en Educación Física: la salud y los juegos modificados. Barcelona: Inde.

Escobar-Pérez, J., \& Cuervo-Martínez, A. (2008). Validez de contenido y juicio de expertos: una aproximación a su utilización. Avances en Medición, 6, 27-36.

Farias, C. F., Mesquita, I. R., \& Hastie, P. A. (2015). Game Performance and Understanding Within a Hybrid Sport Education Season. Journal of Teaching in Physical Education, 34(4), 725-729. doi:10.1123/jtpe.20150189

Feu, S., Ibáñez, S. J., García-Rubio, J., \& Antúnez, A. (2017). La investigación sobre la enseñanza de los deportes de invasión y su transferencia al contexto escolar. Revista Portuguesa de Ciências do Desporto, 17, 50-58. doi:10.5628/rpcd.17.S1A.50

Field, A. (2013). Discovering statistics using SPSS (4 ${ }^{\text {th }}$ Ed.). London: Sage publications Ltd.

Gamonales, J. M., León, K., Muñoz, J., González-Espinosa, S., \& Ibáñez, S. J. (2018). Validación del IOLF5C para la eficacia del lanzamiento en fútbol para ciegos / Validation of IOLF5C for the Efficiency of the Launch in the Football for Blind. Revista Internacional de Medicina y Ciencias de la Actividad Física y el Deporte, 18(70), 361-381. doi:10.15366/rimcafd2018.70.010

García, J. A., y Ruiz, L. M. (2003). Análisis comparativo de dos modelos de intervención en el aprendizaje del balonmano. Revista de Psicología del Deporte, 12(1), 55-66.

García-Ceberino, J. M., Feu, S., \& Ibáñez, S. J. (2019). Comparative Study of Two Intervention Programmes for Teaching Soccer to School-Age Students. Sports, 7, 74, doi:10.3390/sports7030074

García-Ceberino, J. M., Gamero, M. G., Feu, S., Ibáñez, S. J. (2020). Experience as a Determinant of Declarative and Procedural Knowledge in School Football. International Journal of Environmental Research and Public Health, 17, 1063, doi:10.3390/ijerph17031063 
García-Martín, A., Antúnez, A., \& Ibáñez, S. J. (2016). Análisis del proceso formativo en jugadores expertos: validación de instrumento / Analysis of Expert Players' Training Process: Validation of Tools. Revista Internacional de Medicina y Ciencias de la Actividad Física y el Deporte, 16(61), 157-182. doi:10.15366/rimcafd2016.61.012

García-Santos, D., \& Ibáñez, S. J. (2016). Diseño y validación de un instrumento de observación de un árbitro de baloncesto (IOVAB). Sport-TK: Revista Euroamericana de Ciencias del Deporte, 5(2), 15-26. doi:10.6018/264601

George, D., \& Mallery, P. (2003). SPSS for Windows step by step: A simple guide and reference. 11.0 update (4 ${ }^{\text {th }}$ Ed.). Boston: Allyn \& Bacon.

González-Espinosa, S., Ibáñez, S. J., \& Feu, S. (2017a). Diseño de dos programas de enseñanza del baloncesto basados en métodos de enseñanza-aprendizaje diferentes. E-balonmano: Revista de Ciencias del Deporte, 13(2), 131-152.

González-Espinosa, S., Ibáñez, S. J., Feu, S., \& Galatti, L. R. (2017b). Programas de intervención para la enseñanza deportiva en el contexto escolar, PETB y PEAB: Estudio preliminar. Retos: Nuevas Tendencias en Educación Física, Deporte y Recreación, 31, 107-113.

González-Espinosa, S., Mancha-Triguero, D., García-Santos, D., Feu, S., e Ibáñez, S. J. (2019). Diferencia en el aprendizaje del baloncesto según el género y metodología de enseñanza. Revista de Psicología del Deporte, 28(Suppl 1), 86-92.

Gray, S., \& Sproule, J. (2011). Developing pupils' performance in team invasion games. Physical Education and Sport Pedagogy, 16(1), 15-32. doi:10.1080/17408980903535792

Harvey, S., Song, Y., Baek, J. H., \& Van der Mars, H. (2016). Two sides of the same coin: Student physical activity levels during a game-centred soccer unit. European Physical Education Review, 22(4), 411-429. doi:10.1177/1356336X15614783

Ibáñez, S. J. (2008). La planificación y el control del entrenamiento técnicotáctico en Baloncesto. En N. Terrados, \& J. Calleja (Coords.), Fisiología, entrenamiento y medicina del Baloncesto (pp. 299-313). Barcelona: Paidotribo.

lbáñez, S. J. (2009). Planificación de una temporada en la iniciación al baloncesto. En G. Ortega, \& A. C. Jiménez (Coords.), Táctica y técnica en la iniciación al baloncesto (pp.69-99). Sevilla: Wanceulen.

Ibáñez, S. J., Feu, S., \& Cañadas, M. (2016). Sistema integral para el análisis de las tareas de entrenamiento, SIATE, en deportes de invasión. Ebalonmano.com: Revista de Ciencias del Deporte, 12(1), 3-30.

Ibáñez, S. J., Martínez-Fernández, S., González-Espinosa, S., García-Rubio, J., \& Feu, S. (2019). Designing and Validating a Basketball Learning and Performance Assessment Instrument (BALPAI). Frontiers in Psychology, 10, 1595. doi:10.3389/fpsyg.2019.01595

Jiménez, J., Salazar, W., \& Morera, M. (2013). Diseño y validación de un instrumento para la evaluación de patrones básicos de movimiento. Motricidad. European Journal of Human Movement, 31, 87-97. doi:10.22579/20114680.48

Kirk, D. (2005). Model-based teaching and assessment in Physical Education: the Tactical Games Model. En K. Green, \& K. Hardman (Eds.), Physical 
Education: essential issues (pp.128-142). London: Sage. doi:10.4135/9781446215876.n8

König, S., \& Singrün, P. (2013). Wirkungen und Festigkeit von motorischen Lernund Trainingsprozessen im Sportunterricht [Effect and consistency of motor learning and training processes in physical education]. Spectrum der Sportwissenschaften, 25, 4-31.

Launder, A. G., \& Piltz, W. (2006). Beyond «Understanding» to skilful play in games, through Play practice. Journal of Physical Education New Zealand, 39(1), 47-57.

López, V., \& Castejón, F. J. (2005). La enseñanza integrada técnico-táctica de los deportes en edad escolar. Explicación y bases de un modelo. Apunts: Educación Física y Deportes, 79(1), 40-48.

Méndez, A. (2009). Modelos actuales de iniciación deportiva: unidades didácticas sobre deportes de invasión. Sevilla: Wanceulen.

Merino, C., \& Livia, J. (2009). Intervalos de confianza asimétricos para el índice la validez de contenido: Un programa Visual Basic para la $\mathrm{V}$ de Aiken. Anales de Psicología, 25(1), 169-171.

Mesquita, I., Farias, C., \& Hastie, P. (2012). The impact of a hybrid Sport Education-Invasion Games Competence Model soccer unit on students' decision making, skill execution and overall game performance. European Physical Education Review, 18(2), 205-219. doi:10.1177/1356336x12440027

Metzler, M. W. (2011). Instructional Models for Physical Education (3 ${ }^{\text {rd }}$ Ed.). Scottsdale, AZ, USA: Holocomb Hathaway.

Mitchell, S. A., Oslin, J. L., \& Griffin, L. L. (2013). Teaching Sport Concepts and Skills: A Tactical Games Approach for Ages 7 to 18 (3 ${ }^{\text {rd }}$ Ed.). Champaign, IL: Human Kinetics.

Nunnally, J. C. (1978). Psychometric methods. New York: McGraw-Hill.

Nunnally, J. C., \& Bernstein, I. H. (1994). Pychometric theory (3 ${ }^{\text {rd }}$ Ed.). New York: McGraw-Hill.

Ortega, E., Calderón, A., Palao, J., \& Puigcerver, M. (2009). Diseño y validación de contenido de un cuestionario sobre la satisfacción, participación y opinión de mejora en las clases de educación física en secundaria. Wanceulen E.F. Digital, 5, 14-26.

Ortega, E., Jiménez, J., Palao, J., \& Sainz, P. (2008). Diseño y validación de un cuestionario para valorar las preferencias y satisfacciones en jóvenes jugadores de baloncesto. Cuadernos de Psicología del Deporte, 8(2), 3958.

Ortega-Toro, E., García-Angulo, A., Giménez-Egido, J. M., García-Angulo, F. J., \& Palao, J. M. (2019). Design, Validation, and Reliability of an Observation Instrument for Technical and Tactical Actions of the Offense Phase in Soccer. Frontiers in Psychology, 10, 22. doi:10.3389/fpsyg.2019.00022

Penfield, R., \& Giacobbi, P. (2004). Applying a score confidence interval to Aiken's item content-relevance index. Measurement in Physical Education and Exercise Science, 8(4), 213-225. doi:10.1207/s15327841mpee0804_3

Pérez-Muñoz, J., Yagüe, S., \& Sánchez-Sánchez, J. M. (2015). El proceso de enseñanza-aprendizaje a los deportes colectivos. Sevilla: Wanceulen. 
Piéron, M. (1999). Para una enseñanza eficaz de las actividades físicodeportivas. Barcelona: Inde.

Polit, D., \& Hungler, B. (2000). Investigación científica en Ciencias de la Salud (6 $6^{a}$ Ed.). México: McGraw-Hill.

Robles, A., Robles, J., Giménez, F. J., \& Abad, M. T. (2016). Validación de una entrevista para estudiar el proceso formativo de judokas de élite / Validation Of An Interview For Study The Process Of Formation Of Elite Judokas. Revista Internacional de Medicina y Ciencias de la Actividad Física y el Deporte, 16(64), 723-738. doi:10.15366/rimcafd2016.64.007

Rodríguez, G., Gil, J., \& García, E. (1996). Métodos de investigación cualitativa. Málaga: Aljibe.

Rodríguez, J. E., Mato, J. A., \& Pereira, M. C. (2016). Análisis de los métodos tradicionales de enseñanza-aprendizaje de los deportes colectivos en Educación Primaria y propuestas didácticas innovadoras. Sportis Sci J, 2(2), 254-267. doi:10.17979/sportis.2016.2.2.1426

Saénz-López, P. (1997). La Educación Física y su Didáctica. Manual para el profesor. Sevilla: Wanceulen.

Serra, J., García-López, L. M., \& Sánchez-Mora, D. (2011). El juego modificado, recurso metodológico en el fútbol de iniciación. Retos: Nuevas Tendencias en Educación Física, Deporte y Recreación, 20, 37-42.

Smith, L., Harvey, S., Savory, L., Fairclough, S., Kozub, S., \& Kerr, C. (2015). Physical activity levels and motivational responses of boys and girls: a comparison of direct instruction and tactical games models of games teaching in physical education. European Physical Education Review, 21(1), 93-113. doi:10.1177/1356336X14555293

Torres-Luque, G., Fernández-García, A. I., Cabello-Manrique, D., GiménezEgido, J. M., \& Ortega-Toro, E. (2018). Design and Validation of an Observational Instrument for the Technical-Tactical Actions in Singles Tennis. Frontiers in Psychology, 9, 2418. doi:10.3389/fpsyg.2018.02418

Turner, A. P., \& Martinek, T. J. (1999). An investigation into teaching games for understanding: Effects on skill, knowledge, and game play. Research Quarterly for Exercise and Sport, 70(3), 286-296. doi:10.1080/02701367.1999.10608047

Villarejo, D., Ortega, E., Gómez, M. A., \& Palao, J. (2014). Design, validation and reliability of an observational instrument for ball possessions in rugby union. International Journal of Performance Analysis in Sport, 14, 957-969. doi:10.1080/24748668.2014.11868771

Zapatero, J. A. (2017). Beneficios de los estilos de enseñanza y las metodologías centradas en el alumno en educación física. E-balonmano.com: Revista de Ciencias del Deporte, 13(3), 237-250.

Referencias totales / Total references: 67 (100\%)

Referencias propias de la revista / Journal's own references: 3 (4,48\%)

Rev.int.med.cienc.act.fís.deporte - vol. 20 - número 78 - ISSN: 1577-0354 